\title{
Host-based antipoxvirus therapeutic strategies: turning the tables
}

\author{
Anthony S. Fauci and Mark D. Challberg \\ National Institute of Allergy and Infectious Diseases, NIH, Bethesda, Maryland, USA.
}

\begin{abstract}
The potential threat of the smallpox virus as a bioterror weapon has long been recognized, and the need for developing suitable countermeasures has become especially acute following the events of September 2001. Traditional antiviral agents interfere with viral proteins or functions. In a new study, Yang et al. focus instead on host cellular pathways used by the virus. A drug that interferes with the cellular ErbB-1 signal transduction pathway, activated by smallpox growth factor, sheds new light on how the virus replicates in the cell (see the related article beginning on page 379). Drugs that target the ErbB-signaling pathways represent a promising new class of antiviral agents.
\end{abstract}

Developing medical interventions against the threat of bioterrorism has been a part of the national biomedical research agenda at least since the days of the Cold War. However, the immediacy of such a threat was not fully appreciated until the events of the fall of 2001. The attacks on the World Trade Center and the Pentagon and the subsequent release of anthrax spores that infected 22 people and killed 5 transformed a remote possibility into somber reality (1).

Of special concern are category A agents and the need to develop countermeasures against these agents, which are known to cause fatal diseases such as anthrax, smallpox, plague, botulism, tularemia, and viral hemorrhagic fevers (2). The need for countermeasures against the Variola major virus, which causes smallpox, is especially acute because the virus is highly lethal and easily transmitted from person to person and because no effective treatments have yet been approved for use in humans. As a result of the successful global eradication of smallpox in 1977, routine vaccinations have been discontinued, and most people today have little or no immunity against the virus. Concerns about unaccounted-for stocks of smallpox virus surfaced after the fall of the Soviet Union, when it was revealed that massive quantities of the virus had been produced, further heightening the sense of urgency for the development of new therapies (2-4). An

Nonstandard abbreviations used: c-Src, cellular Src; EEV, extracellular-enveloped virus; GF, growth factor; IMV, intracellular mature virus; SPGF, smallpox growth factor; VGF, vaccinia growth factor.

Conflict of interest: The authors have declared that no conflict of interest exists.

Citation for this article: J. Clin. Invest. 115:231-233 (2005). doi:10.1172/JCI200524270. effective smallpox vaccine exists, and several new vaccine candidates are now being tested. Should a deliberate release of smallpox occur, however, it is important to have drug treatments readily accessible to protect against disease and to reduce any adverse effects of a live or attenuated smallpox vaccine. Currently, no such treatments are available, although cidofovir, a nucleoside analog that inhibits viral replication, is being tested clinically for treatment of some poxvirus infections $(5,6)$.

\section{Cl-1033 prevents viral replication in vitro}

Traditional antiviral drugs are generally directed against the proteins and functional pathways of the virus itself. For example, numerous therapies have been developed against HIV that interfere with viral reverse transcriptase, protease, and integrase as well as viral components involved in the binding and fusion of the virus to the target cell (7). However, many viruses evolve rapidly, particularly under selective pressures, and drug resistance almost invariably develops.

Because viruses typically rely upon cellular pathways to self-propagate, another antiviral approach would be to develop drugs that interfere with viral functions that are dependent on the functional machinery of the cell. Such an approach has been adopted by Yang et al., who report in this issue of the JCI (8) on a class of drugs originally developed as anticancer agents that show promise against orthopoxviruses and perhaps other viruses as well.

It has previously been shown that a growth factor (GF) encoded by the genomes of all orthopoxviruses (smallpox growth factor [SPGF] by variola virus, vaccinia growth factor [VGF] by vaccinia virus) binds to and activates the ErbB-1 kinase, a member of the epidermal GF receptor family of tyrosine kinases $(9,10)$. Because the poxvirus-encoded GFs are important for viral pathogenesis $(11,12)$, it seemed likely that inhibiting the cellular GF receptor might be a useful approach to controlling poxvirus infection. Yang et al. now demonstrate that inhibitors of cellular ErbB-1 do in fact disrupt important processes of the viral replication cycle and may represent an important new approach to antiviral chemotherapy (8).

This paper also provides new insight into the role of the poxvirus-encoded GFs in viral pathogenesis, suggesting that poxvirus GFs may play a direct role in virus replication. Previous studies have suggested that VGF acts on cells to stimulate metabolism, thereby increasing the number of cells capable of supporting efficient viral replication (11). Yang et al. (8) examined the effect of the ErbB inhibitor CI-1033 on the growth of variola and vaccinia virus - a smallpoxlike virus - in infected monkey kidney cells in vitro. The drug had no effect on the overall yield of newly made virus in cell culture experiments in which all the cells in the culture were infected simultaneously, but it did have an effect on the appearance of plaques, which arise from the initial infection of a single cell and require local spread of the virus from the infected cell to surrounding uninfected cells. Two distinct forms of infectious virions are produced in poxvirus-infected cells: intracellular mature virus (IMV), which is released only following death and lysis of infected cells, and extracellular-enveloped virus (EEV), which is actively extruded from cells by interaction with actin tails (13) (Figure 1). The release of EEV from infected cells is thought to be the principal mechanism for rapid spread of the virus in the infected host. Yang et al. (8) show that the ErbB inhibitor CI-1033 greatly reduces the release of EEV from cells infected with either vaccinia or variola. This reduction in EEV release is likely due, at least in part, to inhibition of the viral GF activation of ErbB-1 because, even in the absence of CI-1033, deletion of the GF gene from vaccinia virus has an effect 


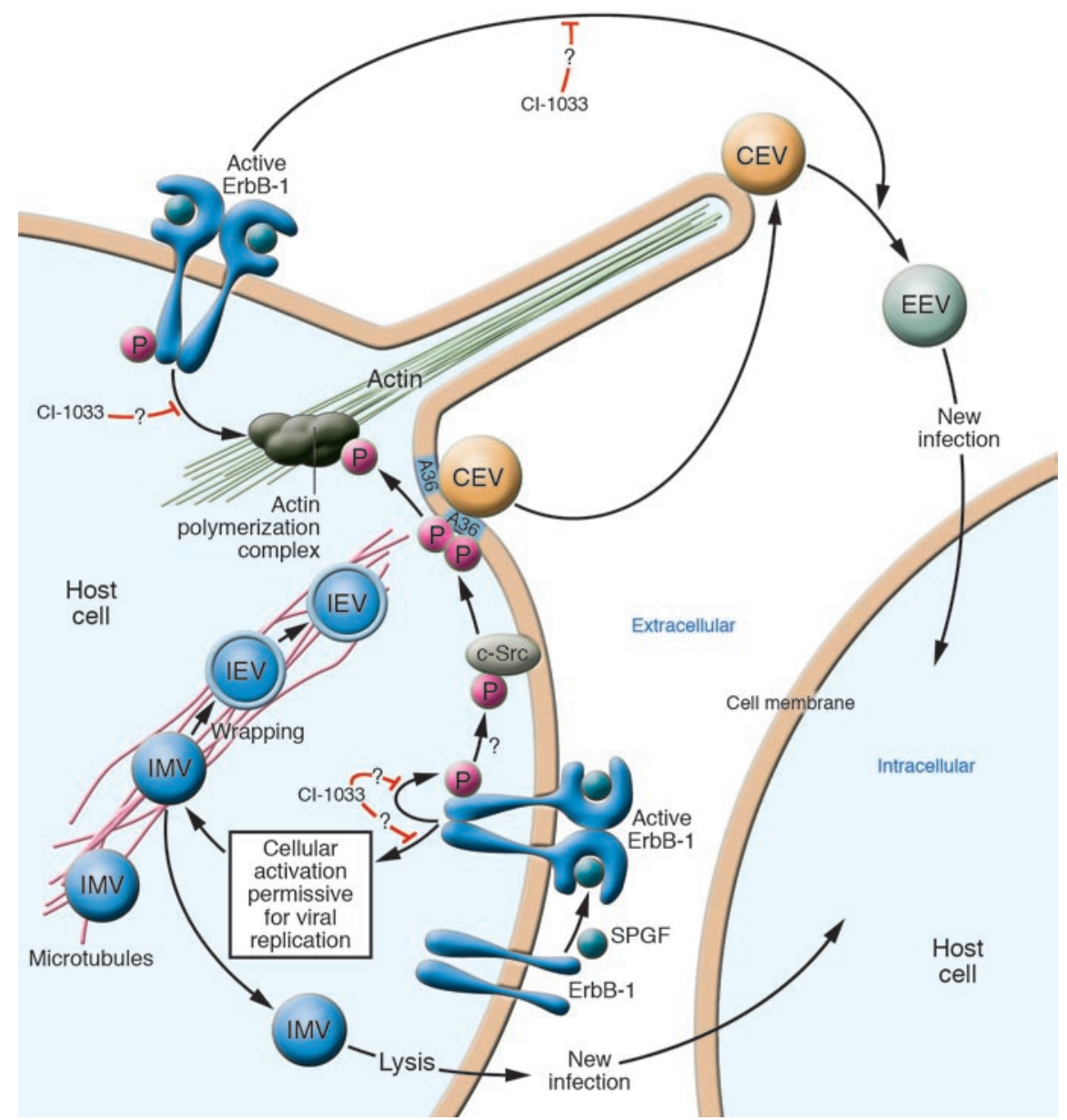

Figure 1

Possible mechanism for SPGF-dependent pathogenesis of variola virus and the inhibitory effect of $\mathrm{Cl}-1033$. SPGF binds to the ErbB-1 receptor, inducing dimerization and activation. Active phosphorylation of relevant receptors, through unknown mechanisms, may render the cell permissive for viral replication. Phosphorylated ErbB-1 can also activate c-Src kinase as well as the actin polymerization complex, which regulates the formation of actin tails within the cytoplasm. c-Src in turn activates the viral A36 protein, an event required for activation of the actin polymerization complex. Viral replication yields IMVs, which are released from the host cell through cell death and lysis. Alternatively, IMV particles are transported via microtubules and wrapped with additional membrane and protein components to form intracellular-enveloped virus (IEV) particles. IEVs are transported to the cell membrane where they fuse with the plasma membrane to become cellular-enveloped virus (CEV) particles and activate the actin polymerization complex. Some CEVs are released from the cell as EEV particles, which rapidly disseminate the infection. $\mathrm{Cl}-1033$ can block the phosphorylation of ErbB-1 and subsequent c-Src activation as well as activation of the actin polymerization complex, which as Yang et al. (8) speculate, may play a role in viral extrusion. $\mathrm{Cl}-1033$ may also interfere with events that render cells permissive for viral replication. Figure adapted with permission from Science (15) and Virus Research (13).

on the release of EEV similar to that of CI-1033 on wild-type virus. However, the drug appears to have additional antiviral effects when added to cells infected with mutant virus as shown by the reduction in the size of plaques. It has recently been shown that actin tail formation, which appears to be involved in EEV release, is regulated by phosphorylation of the viral morphogenesis protein A36 by cellular Src (c-Src) $(14,15)$. disease (8). Mice were first infected with virus and treated with CI-1033 in the presence and absence of a monoclonal antibody that neutralizes the IMV form of infectious virions. At moderate challenge doses of virus (close to the $\mathrm{LD}_{50}$ ), CI-1033 treatment greatly increased animal survival and had a moderate effect on viral load in the lung. A much greater effect of the drug was seen when it was administered in conjunction with monoclonal antibody. This result is consistent with what is known about immunotherapies for poxvirus disease: maximum protection is seen when both EEV and IMV are neutralized $(17,18)$. Interestingly, the combination of CI-1033 and anti-IMV antibody also significantly stimulated $\mathrm{T}$ cell immunity in the infected mice (8). The mechanism of this effect remains to be explored.

\section{A new class of antiviral drugs}

The concept of interfering with the dependence of virus replication on cellular machinery is not new. For years, HIV/AIDS researchers have attempted to blunt viral replication by interfering with the cascade of aberrant activation signals that make the cell permissive for viral replication $(19,20)$. The benefit of using drugs such as cyclosporin and mycophenylate, which suppress immune cell activation, to treat HIV infection remains questionable because of the chronic nature of the disease; however, such an approach may be an effective strategy for treating an acute infection, such as smallpox.

In essence, the approach suggested by Yang et al. (8) may serve to turn the tables on the virus by interfering with the very pathways that are required for viral replication and extrusion. Because it is less likely that such a strategy would allow the virus to develop drug resistance and because different members of a virus family will likely use the same host pathways to propagate, this strategy approaches the concept of a "universal" antiviral therapy, at least within the virus family in question. Indeed, replication of many viruses, including poxviruses, is known to be dependent upon the ErbB class of tyrosine kinases, and the screening for and development of drugs that block this and other classes of receptors should be vigorously pursued. This strategy would be especially useful in developing countermeasures against newly emerging infectious diseases and those that are introduced deliberately, as in a bioterror attack.

Over the past several decades, thousands of promising anticancer drugs, including CI-1033, have been developed by pharmaceutical companies to interfere with GF-mediat- 
ed signal transduction cascades such as those coordinated by the ErbB class of receptor tyrosine kinases $(21,22)$. Yang et al.'s study (8) shows that variola and related viruses are dependent upon some of the same pathways that the host cell uses for growth and development. Inhibitors of the ErbB-1 pathway as well as other cell-signal transduction pathways required for viral replication represent a largely untapped source of potential antiviral drugs and merit further exploration.

\section{Acknowledgments}

The authors wish to thank Nancy Touchette, Gregory Folkers, and Bernard Moss for helpful discussions.

Address correspondence to: Anthony S. Fauci, National Institute of Allergy and Infectious Diseases, NIH, Building 31, Room 7A03, 9000 Rockville Pike, Bethesda, Maryland 20892-2520, USA. Phone: (301) 496-2263; Fax: (301) 496-4409; E-mail: afauci@niaid.nih.gov.

\footnotetext{
1. Lane, H.C., La Montagne, J., and Fauci, A.S. 2001 Bioterrorism: a clear and present danger. Nat. Med. 7:1271-1273.
}

2. [Anonymous]. 2002. NIAID biodefense research agenda for CDC category A agents. NIH Publication No. 03-5308. www.niaid.nih.gov/biodefense/ research/biotresearchagenda.pdf.

3. Mahy, B.W.J. 2003. An overview on the use of a viral pathogen as a bioterrorism agent: why smallpox? Antiviral Res. 57:1-5.

4. Smith, G.L., and McFadden, G. 2002. Smallpox: anything to declare? Nat. Rev. Immunol. 2:521-527.

5. Harrison, S.C., et al. 2004. Discovery of antivirals against smallpox. Proc. Natl. Acad. Sci. U. S. A. 101:11178-11192.

6. Ibarra, V., Blanco, J.R., Oteo, J.A., and Rosel, L. 2000. Efficacy of cidofovir in the treatment of recalcitrant molluscum contagiosum in an AIDS patient. Acta Derm. Venereol. 80:315-316.

7. Greene, W.C. 2004. The brightening future of HIV therapeutics. Nat. Immunol. 5:867-871.

8. Yang, H., et al. 2005. Antiviral chemotherapy facilitates control of poxvirus infections through inhibition of cellular signal transduction. J. Clin. Invest. 115:379-387. doi:10.1172/JCI200523220.

9. Kim, M., et al. 2004. Biochemical and functional analysis of smallpox growth factor (SPGF) and anti-SPGF monoclonal antibodies. J. Biol. Chem. 279:25838-25848.

10. Tzahar, E., et al. 1998. Pathogenic poxviruses reveal viral strategies to exploit the ErbB signaling network. EMBO J. 17:5948-5963.

11. Buller, R.M.L, Chakrabarti, S., Cooper, J.A., Twardzik, D.R., and Moss, B. 1988. Deletion of the vaccinia virus growth factor gene reduces virus virulence. J. Virol. 62:866-874.

12. Opgenorth, A., Nation, N., Graham, K., and McFadden, G. 1993. Transforming growth factor alpha,
Shope fibroma growth factor, and vaccinia growth factor can replace myxoma growth factor in the induction of myxomatosis in rabbits. Virology. 192:701-709.

13. Smith, G.L., and Law, M. 2004. The exit of Vaccinia virus from infected cells. Virus Res. 106:189-197.

14. Newsome, T.P., Scaplehorn, N., and Way, M. 2004. Src mediates a switch from microtubule- to actin-based motility of Vaccinia virus. Science. 306:124-129.

15. Hall, A. 2004. Src launches vaccinia. Science. 306:65-67.

16. Yarden, Y., and Sliwkowski, M.X. 2001. Untangling the ErbB signalling network. Nat. Rev. Mol. Cell. Biol. 2:127-137.

17. Hooper,J.W., Custer, D.M., and Thompson, E. 2003. Four-gene-combination DNA vaccine protects mice against a lethal vaccinia virus challenge and elicits appropriate antibody responses in nonhuman primates. Virology. 306:181-195.

18. Fogg, C., et al. 2004. Protective immunity to vaccinia virus induced by vaccination with multiple recombinant outer membrane proteins of intracellular and extracellular virions. J. Virol. 78:10230-10237.

19. Rizzardi, C.P., et al. 2002. Treatment of primary HIV-1 infection with cyclosporin A coupled with highly active antiretroviral therapy. J. Clin. Invest. 109:681-688. doi:10.1172/JCI200214522.

20. Chapuis, A.G., et al. 2000. Effects of mycophenolic acid on human immunodeficiency virus infection in vitro and in vivo. Nat. Med. 6:762-768.

21. Cockerill, G.S., and Lackey, K.E. 2002. Small molecule inhibitors of the class I receptor tyrosine kinase family. Curr. Top. Med. Chem. 2:1001-1010.

22. Wakeling, A.E. 2002. Epidermal growth factor receptor tyrosine kinase inhibitors. Curr. Opin. Pharmacol. 2:382-387.

\section{Knock your SOCS off!}

\section{Derek LeRoith ${ }^{1}$ and Peter Nissley}

1Diabetes Branch, National Institute of Diabetes and Digestive and Kidney Diseases (NIDDK) and

2Metabolism Branch, National Cancer Institute, NIH, Bethesda, Maryland, USA.

The growth hormone/IGF-1-signaling (GH/IGF-1-signaling) system is involved in numerous physiological processes during normal growth and development and also in the aging process. Understanding the regulation of this system is therefore of importance to the biologist. Studies conducted over the past decade have shown that the JAK/STAT pathways are involved in GH signaling to the nucleus. More recently, evidence has been presented that a member of the SOCS family, SOCS2, is a negative regulator of GH signaling. This story began several years ago with the dramatic demonstration of gigantism in the SOCS2-knockout mouse. A more specific definition of the role of SOCS2 in GH signaling is provided in this issue of the JCI (see the related article beginning on page 397) by the demonstration that the overgrowth phenotype of the SOCS2 ${ }^{-/-}$mouse is dependent upon the presence of endogenous GH and that administration of GH to mice lacking both endogenous GH and SOCS2 produced excessive growth.

Nonstandard abbreviations used: $\mathrm{GH}$, growth hormone; GHR, GH receptor; IGF-1R, IGF-1 receptor; PIAS, protein inhibitor of activated STAT; SHP1, Src homology 1-containing tyrosine phosphatase.

Conflict of interest: The authors have declared that no conflict of interest exists.

Citation for this article: J. Clin. Invest. 115:233-236 (2005). doi:10.1172/JCI200524228.
Cytokines regulate an array of biological processes by activating cell surface receptor complexes, a process that initially involves oligomerization and activation of the JAK family of tyrosine kinases. In turn, JAKs phosphorylate the cell surface receptor, and signaling proteins such as STATs are recruited to these phosphotyrosine sites on the receptor; the proximity of the STATs allows them to be phosphorylated by the JAKs (Figure 1). Dimerization of the phosphorylated STATs leads to nuclear migration and regulation of gene expression (1). To control excessive cytokine effects, the cytokine signal is negatively regulated by a number of proteins, including protein tyrosine phosphatases such as Src homology 1-containing tyrosine phosphatase (SHP1), protein inhibitor of activated STAT (PIAS), and SOCS (2). The latter family is comprised of cytokine-inducible $\mathrm{SH} 2$-containing protein and SOCS1-SOCS7. SOCSs are furthermore induced by cytokine signaling and therefore form a closed-loop, negativefeedback control mechanism (Figure 1).

\section{SOCS2, a new player in growth} hormone receptor signaling

While cytokines and their receptors have traditionally been the domain of immunol- 